Accepted for publication in ApJ

\title{
Constraining the Physical Parameters of the Circumstellar Disk OF $\chi$ OPHIUCHI
}

\author{
C. Tycner,${ }^{1}$ C. E. Jones, ${ }^{2}$ T. A. A. Sigut,${ }^{2}$ H. R. Schmitt,${ }^{3,4}$ J. A. Benson, ${ }^{5}$ D. J. Hutter ${ }^{5}$ \\ R. T. Zavala ${ }^{5}$
}

\begin{abstract}
We present a numerical model describing a circularly symmetric gaseous disk around the Be star $\chi$ Ophiuchi. The model is constrained by long-baseline interferometric observations that are sensitive to the $\mathrm{H} \alpha$ Balmer line emission from the disk. For the first time our interferometric observations spatially resolve the inner region of the circumstellar disk around $\chi$ Ophiuchi and we use these results to place a constraint on the physical extent of the $\mathrm{H} \alpha$-emitting region. We demonstrate how this in turn results in very specific constraints on the parameters that describe the variation of the gas density as a function of radial distance from the central star.
\end{abstract}

Subject headings: stars: emission-line, Be — stars: individual ( $\chi$ Oph $)$ - techniques: interferometric

\section{Introduction}

Models of the circumstellar gas around some B-type stars have been constructed for a number of years. Because the recombination in the circumstellar gas produces a series

\footnotetext{
${ }^{1}$ Department of Physics, Central Michigan University, Mt. Pleasant, MI 48859

${ }^{2}$ Department of Physics and Astronomy, The University of Western Ontario, London, Ontario, N6A 3K7, Canada

${ }^{3}$ Naval Research Laboratory, Remote Sensing Division, Code 7215, 4555 Overlook Ave. SW, Washington, DC 20375

${ }^{4}$ Interferometrics, Inc., 13454 Sunrise Valley Drive, Suite 240, Herndon, VA 20171

${ }^{5}$ US Naval Observatory, Flagstaff Station, 10391 W. Naval Observatory Rd., Flagstaff, AZ 86001
} 
of emission lines, most commonly in the hydrogen Balmer series, such systems are usually denoted B-emission or Be stars for short. Models describing the geometry of this circumstellar material have varied from spherical shells (Gehrz et al. 1974), to slabs with constant thickness (Kastner \& Mazzali 1989), to disks with constant opening angles (Waters 1986), to disks with an exponential density fall-off in the direction perpendicular to the plane of the disk (Marlborough 1969). Although the first models assumed a constant temperature throughout the disk, more recent models, such as the ones developed by Millar \& Marlborough (1998, 1999), Carciofi \& Bjorkman (2006), and Sigut \& Jones (2007), obtain a self-consistent temperature throughout the disk by enforcing radiative equilibrium (namely, by balancing the microscopic heating and cooling rates operating within the disk).

While the temperature structure of Be star disks is now the subject of sophisticated modeling, the density structure largely remains ad-hoc with simple, parameterized models. Perhaps the most popular is a power-law density decrease with radius in the equatorial plane coupled with the aforementioned exponential drop perpendicular to the disk. This vertical density structure is assumed to result from the gravitational equilibrium set by the balance between the gradient in the gas pressure and the vertical component of the star's gravitational acceleration. Despite the simplicity of such models, they have been quite successful in matching a wide range of observational diagnostics that are sensitive to the geometric distribution of the circumstellar gas. These include the infrared excess exhibited by Be stars over normal B stars of the same spectral class (Dougherty 1994), the observed continuum linear polarization of Be stars (Wood et al. 1997), and the shapes of the emission lines observed in Be star spectra (see for example Hummel 2000). All of these diagnostics rely only on spatially unresolved spectra or spectropolarimetry. However, even more powerful constraints on the geometry of the emitting regions in Be stars can be obtained by combining these spatially unresolved diagnostics with new optical interferometric observations that directly resolve the structure of the emitting region on the sky Quirrenbach et al. 1997; Tycner et al. 2006b; Gies et al. 2007).

To demonstrate how specific constraints can be placed on a disk model using observations that spatially resolve the source, we chose to concentrate on the Be star $\chi$ Oph (B2Ve; HR 6118; HD 148184). This relatively nearby star with a Hipparcos distance of $150 \pm 17$ pc (Perryman et al. 1997) has a strong $\mathrm{H} \alpha$ emission and therefore is an ideal candidate for probing the disk structure at these wavelengths. 


\section{Observations}

\subsection{Interferometry}

We acquired interferometric observations of $\chi$ Oph on four nights between 2006 June 11 and 2006 June 18. All observations were made with the same instrumental configuration. We used 4 different telescopes, resulting in five unique baselines, with one baseline signal measured twice on two independent output beams (see Fig. 2 in Tycner et al. 2006b, for a schematic of the beam combiner). The baselines used and their corresponding lengths are: AC-AE (18.9 m), AC-AW (22.2 m), AW-W7 (29.5 m), AC-W7 (51.6 m), AE-W7 $(64.4 \mathrm{~m})$, with the AC-W7 baseline measured on two output beams. The observations were obtained using two spectrographs, each recording simultaneously signal from 3 baselines at 16 spectral channels in the wavelength range 560-870 $\mathrm{nm}$. The resulting $(u, v)$-plane coverage of the interferometric observations obtained from the spectral channel containing the $\mathrm{H} \alpha$ emission line is shown in Figure 1.

The observations of $\chi$ Oph were interleaved with observations of a nearby calibrator star $\zeta$ Oph (O9V; HR 6175; HD 149757). The choice of the calibrator star was based on the relative proximity on the sky to the target and the spectral type, which was similar to that of the target star. Furthermore, the angular diameter of $\zeta$ Oph has been directly measured by Hanbury Brown et al. (1974) using an intensity interferometer with sufficient accuracy to be competitive with other methods that estimate angular diameters based on theoretical or empirical models. Therefore, for the calibrator star, we adopted a uniform disk angular diameter of $0.50 \pm 0.05$ mas as obtained by Hanbury Brown et al. (1974).

The data reductions followed standard NPOI procedures and will only briefly be described here. The raw data are processed to produce squared visibilities $\left(V^{2}\right)$ averaged into $1 \mathrm{~s}$ intervals. These data are then flagged to eliminate points with fringe tracking and pointing problems (Hummel et al. 1998, 2003). The flagged data set is then used to obtain $30 \mathrm{~s}$ averages (we refer to these averages as scans), which are bias corrected using data obtained off the fringe (these are also known as incoherent scans). Table 1 lists the individual nights and the number of scans acquired on each night. Lastly, the squared visibilities from the spectral channel containing the $\mathrm{H} \alpha$ emission line are calibrated with respect to the continuum channels (see Tycner et al. 2003, 2006a, for a detailed description of the procedures). Our final data set (shown in Fig. 2) consists of the ensemble of all the $V^{2}$ data from the $\mathrm{H} \alpha$ channel obtained on all four nights listed in Table 1, The individual calibrated $\mathrm{H} \alpha V^{2}$ values

are also listed in Table 2, Because squared visibilities represent the normalized Fourier power of the source structure on the sky, the interferometric observations can be compared directly to the Fourier transform of the synthetic image produced by the model. 
The observations of $\chi$ Oph used in this study were not optimized for the purpose of calibrating with respect to an external calibrator. However, such a calibration allows one to inspect the continuum channels for the signature of a resolved stellar disk or a binary companion. Using the scans obtained on $\zeta$ Oph to obtain the instrumental system response, we found that large systematic residuals from scan to scan remained after the calibration, which we attribute to atmospheric variations on a timescale of minutes that are poorly sampled by the scans on the calibrator. For this reason, we can only conclude that the central star at the continuum channels is marginally resolved with a uniform disk diameter of $\lesssim 1$ mas. An interferometric signature of a resolved binary is less susceptible to systematic variations from scan to scan because of its distinct sinusoidal functional form that can be seen across different spectral channels. In fact, the NPOI has been used successfully to detect binaries over a wide range of separations (see for example Hutter et al. 2004). The search for a binary signature in observations of $\chi$ Oph, however, did not yield any convincing evidence of such a signature in our interferometric observations that would correspond to a magnitude difference $(\Delta m)$ of $\sim 3$ or less at separations of few hundred milli-arcseconds (mas) or less. Although we cannot rule out a fainter companion, such a companion would not contribute significantly to the total emission from the system and therefore for the purpose of the analysis presented in this study, $\chi$ Oph can be treated as a single star. It should be noted, however, that $\chi$ Oph has been classified as a single-lined spectroscopic binary by more than one study (Abt \& Levy 1978; Harmaned 1987; Levato et al. 1987), although there is no

agreement on the period of the binary, which was estimated to be $138.8 \mathrm{~d}$ by Abt \& Levy (1978) and 34.1 d by Harmaned (1987).

\subsection{Spectroscopy}

To estimate the strength of the $\mathrm{H} \alpha$-emission in $\chi \mathrm{Oph}$ at the time of interferometric observations we obtained a high resolution spectrum in the $\mathrm{H} \alpha$ region using a fiber-fed Echelle spectrograph at the Lowell Observatory's John S. Hall telescope. The spectrum was acquired on 2006 June 10, just one day before our one week long interferometric run. The spectroscopic data were processed using standard routines developed specifically for the instrument used to acquire the observations (Hall et al. 1994). The final reduced spectrum in the $\mathrm{H} \alpha$ region reaches a resolving power of 10,000 and a signal-to-noise ratio of few hundred (see Fig. 3).

In addition to the spectrum shown in Figure 3 we have also acquired spectroscopic observations of $\chi$ Oph in 2006 May and 2006 September. The peak intensities and the equivalent widths (EWs) of the $\mathrm{H} \alpha$ emission line all agree to within $1 \%$ with the line profile 
shown in Figure 3. Therefore, we assume that during our interferometric run the overall emission in the $\mathrm{H} \alpha$ line was stable and combining all of the interferometric observations into one data set is justified.

\section{Determining the Size of the $\mathbf{H} \alpha$-emitting Region}

We followed the procedure described by Tycner et al. (2006b) to determine the angular extent of the $\mathrm{H} \alpha$ disk. We fitted a circularly symmetric Gaussian model to the interferometric data and obtained a best-fit diameter (defined as full-width at half-maximum; FWHM) for the disk of $3.46 \pm 0.07$ mas, which at a distance of $150 \mathrm{pc}$ corresponds to a diameter of $0.52 \mathrm{AU}\left(112 R_{\odot}\right)$. The fit results in a reduced $\chi^{2}$ value of 0.95 , and therefore, there is no indication that the observational signature deviates from circular symmetry or a Gaussian shape for that matter (shown as dash-dotted line in Fig. 22). In fact, this is the best observational evidence to date that a Gaussian model is not only the simplest mathematical description of the radial distribution of the $\mathrm{H} \alpha$ emission in a circumstellar disk, but it is also fully consistent with the observed distribution. This is in addition to similar conclusions obtained previously by Tycner et al. (2006b) for two other Be stars, $\gamma$ Cas and $\phi$ Per.

It is interesting to compare our results based on the Gaussian model fit with the simple estimator based on $\mathrm{H} \alpha$ EW obtained by Grundstrom \& Gies (2006). With the stellar parameters for $\chi$ Oph listed in Table 3 and assuming that the disk is viewed at $20^{\circ}$ (see $\S 5.2$ ), for an EW of $-7.1 \mathrm{~nm}$ based on our spectroscopic observations Grundstrom \& Gies (2006) predict 1 a disk radius of $9.2 R_{\star}$. This agrees well with the radius of $\approx 9.8 R_{\star}$ we obtain based on the Gaussian fit (using half of our FWHM measure).

Although characterizing the $\mathrm{H} \alpha$ emitting region with a Gaussian model can be a useful tool, especially when only a limited interferometric data set is available to constrain the disk characteristics, it is only a simple observational parameterization. However, in cases where the interferometric data set covers wide range of spatial frequencies it is desirable to extract more information about the physical characteristics of the disk, such as density and temperature distribution. For this purpose a much more sophisticated model is needed.

\footnotetext{
${ }^{1}$ We have used the programs provided by the authors at http://www.chara.gsu.edu/ gies/Idlpro/BeDisk.tar to extrapolate for equivalent widths beyond the values considered in Fig. 1 of Grundstrom \& Gies (2006).
} 


\section{Disk Model}

The radiative equilibrium model used to represent the thermal structure of the gaseous circumstellar disk surrounding $\chi$ Oph has been computed using the BEDISK code, which is described in detail by Sigut \& Jones (2007). This code incorporates many improvements over previous treatments, most notably the use of a solar chemical composition for the circumstellar gas, which is an important ingredient for calculating heating and cooling rates. These models are ideally suited to compare with interferometric observations because model monochromatic, 2-dimensional images of the circumstellar disk at specific wavelengths can be computed. The BEDISK code can also be used to compute the hydrogen line spectra and the overall spectral energy distribution. For the calculation of the hydrogen line profiles, the disk is assumed to be in pure Keplerian rotation. There is considerable evidence that Be star disks are indeed rotationally supported based on the detailed analysis of line profiles (Hummel \& Vrancken 2000) and on the interpretation of V/R variations as one-armed density waves in the disk, which are predicted for Keplerian disks (Okazaki 2007). Recent work based on long-baseline interferometry has also provided observational evidence in support of Keplerian rotation (Meilland et al. 2007).

The disk density model of Sigut \& Jones (2007) requires only a few input stellar and disk parameters. It is assumed that the density in the equatorial plane of the disk is given by an $R^{-n}$ power-law in radial distance (where $R$ is the distance from the star's rotation axis), and that the density distribution vertical to the equatorial plane (in the $Z$ direction) is set by the hydrostatic equilibrium established by the gas pressure gradient and the vertical component of the star's gravitational acceleration. Such a model generally produces a thin disk in which $Z / R \ll 1$, and in this case the form of the density distribution is particularly simple:

$$
\rho(R, Z)=\rho_{0}\left(\frac{R_{\star}}{R}\right)^{n} e^{-\left(\frac{Z}{H}\right)^{2}},
$$

where $\rho_{0}$ is the density at the inner edge of the disk in the equatorial plane, $n$ is the index in the radial power-law, and $H$ is the scale height in the $Z$ direction and is given by

$$
H=\sqrt{\frac{2 R^{3}}{\alpha_{0}}}
$$

with the parameter $\alpha_{0}$ of the form:

$$
\alpha_{0}=G M_{\star} \frac{\mu_{0} m_{\mathrm{H}}}{k T_{0}} .
$$

In these expressions, $M_{\star}$ and $R_{\star}$ are the stellar mass and radius, respectively; $T_{0}$ and $\mu_{0}$ are the assumed (vertically isothermal) temperature and mean-molecular weight at the radial 
distance $R$. This simple, analytical form for the density is possible because it is assumed that the vertical pressure scale height at each $R$ can be represented by a single temperature $T_{0}$. Although this assumption might not be directly applicable to dense disks where strong vertical temperature gradients in the disk might be present, Sigut et al. (2007) examined the accuracy of this assumption in detail (in pure hydrogen disks) and found that in most cases, the differences in typical predicted diagnostics, such as the $\mathrm{H} \alpha$ profiles and IR excesses, are not large for models in consistent radiative and (vertical) hydrostatic equilibrium if the density-averaged disk temperature is used for the parameter $T_{0}$. The largest variations are found for the densest models (with largest $\rho_{0}$ ) as these models develop a cool, equatorial zone close to the star. Hence for this current work, we have adopted the simpler density structure given by equation 1 with the parameter $T_{0}$ being constant for all values of $R$ and $Z$. In the case of models constructed for $\chi$ Oph we set $T_{0}$ at $10,500 \mathrm{~K}$ based on typical density-weighted disk temperatures obtained for models in reasonable agreement with the observations of $\chi$ Oph.

With this model for the disk gas density the parameters $\rho_{0}$ and $n$ are free to be varied to match the interferometric visibilities and $\mathrm{H} \alpha$ line profile of $\chi$ Oph. Before we can obtain a self-consistent solution for a disk model with specific $\rho_{0}$ and $n$ values, we need to adopt the parameters for the central star. The reported $T_{\text {eff values for } \chi}$ Oph in the literature range from the low of $18,000 \mathrm{~K}$ to a high of 29,600 K (Gorava 1984; Waters 1986; Frémat et al. 2005; Zorec et al. 2005). Furthermore, Goraya (1984) not only detected variations in visual magnitude of $\chi$ Oph but also reported that such variations were accompanied by changes in the slope of the continuum, resulting in variations of derived $T_{\text {eff }}$ values by more than $4,000 \mathrm{~K}$. Because we do not attempt to model the intrinsic variability of the source in this study, we adopt $T_{\text {eff }}$ based on the spectral type of $\chi$ Oph, a main sequence B2 star, which based on tabulations of de Jager \& Nieuwenhuijzen (1987) gives a $T_{\text {eff }}$ of 20,900 K. The stellar mass and radius are less critical parameters for the disk models and we adopted the average values based on the spectral type tabulated by $\operatorname{Cox}(2000)$ of $10.9 M_{\odot}$ and $5.7 R_{\odot}$. The model input parameters for the stellar component are listed in Table 3 .

Figure 4 shows the thermal structure of a disk model with $n=2.5$ and $\rho_{0}=2 \times 10^{-11} \mathrm{~g}$ $\mathrm{cm}^{-3}$ as a function of radial distance from the central star and distance from the equatorial plane. Although the computational grid extends out to $285 R_{\odot}\left(50 R_{\star}\right)$ from the central star, the figure shows only the region out to $200 R_{\odot}$. There is a conspicuous cooler region in the equatorial plane of the disk within the first $\sim 50 R_{\odot}$ from the central star. This cooler volume represents the region of the disk where the density and the corresponding optical depths are larger. The disk temperature reaches a minimum of $\approx 6,000 \mathrm{~K}$ in this volume. The density perpendicular to the equatorial plane falls off approximately exponentially, so regions surrounding this cool volume have significantly lower densities and are optically thin 
resulting in greater temperatures. These features in the disk thermal structure are typical of other models presented in the literature for Be stars with moderate to high disk densities (for

example see Jones, Sigut \& Marlborough 2004; Carciofi \& Bjorkman 2006; Sigut \& Jones 2007). The overall average temperature of the disk over the computation grid is $\approx 10,400 \mathrm{~K}$, which is consistent with our adopted temperature used in the vertical hydrostatic equilibrium calculations (recall $T_{0}$ ). It should also be noted that the temperature structure shown in Figure 4 is influenced by the incoming radiation from the central star and therefore ultimately the thermal structure will be affected by the choice of stellar parameters, such as $T_{\text {eff }}$ and $R_{\star}$.

\section{Constraining the Model}

\subsection{Synthetic $\mathbf{H} \alpha$ Image}

A self-consistent temperature solution for the disk density model (like that shown in Fig. 4) can be used to directly compute the specific intensity of radiation emitted perpendicular to the plane of the disk. As the hydrogen level populations are found naturally as part of the thermal solution, the $\mathrm{H} \alpha$ line emissivity and opacity,

$$
\eta_{\nu}^{32}=\frac{h \nu_{23}}{4 \pi} N_{3} A_{32} \phi_{\nu}
$$

and

$$
\chi_{\nu}^{23}=\frac{h \nu_{23}}{4 \pi}\left(N_{2} B_{23}-N_{3} B_{32}\right) \phi_{\nu}
$$

are known at each $(R, Z)$ location in the disk. Here $N_{2}$ and $N_{3}$ are the hydrogen number densities in levels 2 and 3 respectively, $B_{23}, B_{32}$ and $A_{32}$ are the Einstein probability coefficients for $\mathrm{H} \alpha$, and $\phi_{\nu}$ is the $\mathrm{H} \alpha$ line profile. For the latter, we have adopted the routines of Barklem \& Piskunov (2003) which include the contributions of (thermal) Doppler broadening and collisional broadening (including the linear Stark effect). Turbulent (or microturbulent) broadening was not considered.

To form the total opacity and emissivity at the frequency of $\mathrm{H} \alpha$, we have added the continuous free-free and bound-free emissivity and opacity of hydrogen to equations 4 and 5 . We have also included electron scattering as a coherent source of opacity but not as a source of emissivity. In a radiative equilibrium (or thermal-balance) calculation it is not usual to include continuum scattering as it leads to no net energy gain or loss by the gas. However, non-coherent electron scattering is capable of broadening spectral lines and blurring continuum edges (Rybicki \& Hummer 1994), and this process could potentially effect the $\mathrm{H} \alpha$ EW measure. Although these effects are ignored in the current study, non-coherent electron 
scattering will be included in future work. Given the opacity and emissivity, we solve the equation of radiative transfer at each radial distance $R$ in the disk along a ray in the $Z$ direction, i.e.,

$$
\frac{d I_{\nu}(R, Z)}{d Z}=\eta_{\nu}^{32}-\chi_{\nu}^{23} I_{\nu}(R, Z)
$$

The formal solution was performed with the short-characteristics method of Olson \& Kunasz (1987). This gives the specific intensity emerging perpendicular to the disk and is what an external observer would see if the disk was viewed at $i=0^{\circ}$.

To obtain a profile that can be compared with observations, the kinematic broadening of the disk's (assumed) Keplerian rotation must be included. As we estimate that the inclination of the rotation axis of $\chi \mathrm{Oph}$ is quite small $\left(i \approx 20^{\circ}\right.$, see below), we have assumed that the specific intensity at this small inclination is well approximated by the $i=0^{\circ}$ intensities computed above. Given this, we simply shift the $i=0^{\circ}$ spectrum of each projected area of the disk and star on the sky by its radial velocity. For areas on the stellar surface, we have adopted the photospheric H $\alpha$ profiles computed in LTE by the code of Barklem \& Piskunov (2003). The radial velocity of each patch on the stellar surface followed from the assumption of a spherical star in solid body rotation with an equatorial velocity of $375 \mathrm{~km} \mathrm{~s}^{-1}$ (giving a $v \sin i$ of $144 \mathrm{~km} \mathrm{~s}^{-1}$; see below). Clearly this procedure is not appropriate for large inclination angles as the perpendicular rays through the disk would no longer be representative of the rays in the direction towards the external observer. However, direct comparison of the above procedure with a complete routine that solves the transfer equation along the inclined rays pointing at the observer shows that the simpler procedure gives good results for small inclinations and the approximate procedure is computationally much faster.

We specify more than a hundred wavelength steps to cover the $15 \mathrm{~nm}$ wide spectral region centered at the $\mathrm{H} \alpha$ line, which corresponds to the spectral channel used to obtain the interferometric observations. The integrated net intensity over that $15 \mathrm{~nm}$ spectral window is shown in Figure 5 where clearly the total intensity is dominated by the bright central star. This is expected because the $15 \mathrm{~nm}$ wide spectral region is much wider than the $\mathrm{H} \alpha$ emission line (recall Fig. 3) and therefore the $\mathrm{H} \alpha$ channel receives significant fraction of the light from the central star. In turn, this radial intensity distribution can be used to construct a circularly symmetric image that corresponds to the total intensity in the $\mathrm{H} \alpha$ channel.

The relatively small $v \sin i$ value reported for $\chi$ Oph of $144 \mathrm{~km} \mathrm{~s}^{-1}$ by Zorec et al. (2005) and the lack of interferometric signature that would suggest large deviations from circular symmetry (recall (3), both suggest that the inclination angle of the disk to the plane of the sky is small. Assuming that the star is rotating near its critical velocity of $477 \mathrm{~km} \mathrm{~s}^{-1}$ (estimated based on its spectral type; see Porter 1996, and references therein), the inclination angle $i$ must be $\sim 18^{\circ}$ or more for the star not to rotate above its critical velocity. 
As rapid rotation is a known characteristic of Be stars, we adopt $20^{\circ}$ for the inclination angle of $\chi$ Oph. Furthermore, because the axial ratio produced by the projection effect on geometrically thin and circularly symmetric disks scales as a cosine of the inclination angle, an inclination of $20^{\circ}$ produces only a $\sim 6 \%$ departure from circular symmetry (i.e., an axial ratio of 0.94). For circumstellar disks with non-negligible opening angles the effect would be even smaller. However, an axial ratio of 0.94 would be undetectable in our data because it affects the $V^{2}$ values at a level much smaller than the precision of our interferometric observations (compare the dash-dotted and dotted curves in Fig. 2). Therefore, for the purpose of this study we will treat the synthetic images as circularly symmetric and will ignore the small projection effect on the images.

The squared visibilities from the $\mathrm{H} \alpha$ channel measure the normalized Fourier power of the source structure on the sky, and therefore the Fourier transform of the model image and the interferometric observations can be directly compared. To accomplish this we first construct a circularly symmetric image corresponding to the radial distribution of the integrated intensity over the $15 \mathrm{~nm}$ wide spectral bandpass covered by the NPOI spectral channel containing the $\mathrm{H} \alpha$ emission line. Because the model intensities are calculated for radial region extending up to $50 R_{\star}$ from the central star, we require minimum dimensions of the model image of $100 R_{\star} \times 100 R_{\star}$ to fully describe the output. However, we extend our synthetic image to $1000 R_{\star} \times 1000 R_{\star}$ dimensions (the outer regions are filled with zeros) to increase the sampling frequency of the model in the Fourier space. The image is sampled every $0.2 R_{\star}$ and this is sufficient to avoid any aliasing problems at the high spatial frequencies covered by the observations. The region of interest of the synthetic image is shown in Figure 6, The 2-D Fourier transform of this circularly symmetric image results in a function that also has circular symmetry. The normalized Fourier power of this transform can then be plotted as a function of the radial spatial frequency (shown as a solid line in Fig. 22). We use the standard $\chi^{2}$ statistic to assess the goodness-of-fit of the model to the actual data obtained on the source.

\subsection{The Parameter Space}

We explore the parametric region of of the disk density model (eq. 1) by computing over 500 parameter pairs of $n$ and $\rho_{0}$. We covered the range of $1.8-5.3$ in $n$ and adjusted $\rho_{0}$ from

a low of $1.0 \times 10^{-12} \mathrm{~g} \mathrm{~cm}^{-3}$ to a high of $8.0 \times 10^{-8} \mathrm{~g} \mathrm{~cm}^{-3}$. The majority of the solutions were completely inconsistent with the interferometric observations, and those solutions that yielded reduced $\chi^{2}$ value of 7 or less were concentrated in a very well defined 'valley' in the $\chi^{2}$ space (see Fig. 7). 
The model that results in the lowest reduced $\chi^{2}$ value of 1.17 corresponds to $n$ of 2.5 and

$\rho_{0}$ of $2.0 \times 10^{-11} \mathrm{~g} \mathrm{~cm}^{-3}$. The thermal structure (Fig. 4), $\mathrm{H} \alpha$ model image (Fig. 6), and the model curve shown in Figure 2 all correspond to this best-fit model. Furthermore, assuming that the disk is viewed at an inclination angle of $20^{\circ}$ (as discussed in $\S$ 5.1), we obtain an excellent agreement with the observed $\mathrm{H} \alpha$ line profile obtained through spectroscopy (see Fig. 3). In fact, for the disk model defined by our best-fit to interferometric data, the $\mathrm{H} \alpha$ profile can only be reproduced assuming $i$ of $20^{\circ}$. For any smaller inclination the model profile is simply too sharp (due to the decreased rotational broadening) and for larger values of $i$ the line becomes too broad and weaker than the actual observed profile.

In addition to the best-fit solution found at $n$ of 2.5 and $\rho_{0}$ of $2.0 \times 10^{-11} \mathrm{~g} \mathrm{~cm}^{-3}$ it is clearly evident from Figure 7 that there exists a range of parameter values in the $\chi^{2}$ space that produce disk models that fit the observational data acceptably well. However, these solutions appear to be confined to a very narrow range in the $\chi^{2}$ space. We attribute this range of solutions to the changing total $\mathrm{H} \alpha$ flux generated by a model, where most of the solutions in the upper-left part of the figure produce too much $\mathrm{H} \alpha$ flux with respect to the central star and those in the lower-right part of the figure do not produce enough $\mathrm{H} \alpha$ flux. These effects can be related to different disk thermal structures and different densities, which in turn will affect the apparent sizes of the $\mathrm{H} \alpha$-emitting regions. A linear least-squares fit to solutions with reduced $\chi^{2}<7$ in the $\log \rho_{0}$ versus $n$ plane yields a slope of $1.18 \pm 0.03$ (and an intercept of $-13.6 \pm 0.1$ ), where the uncertainty does not account for the effects of gridding or the choice of the reduced $\chi^{2}$ cutoff value. Nevertheless, this indicates that at least for a range of $n$ values between 2.5 and 4.0 the disk solutions that produce interferometric signatures closest to the observational data fall along a relation where $\log \rho_{0}$ is directly proportional to

$n$. This is in agreement with similar conclusions made by Gies et al. (2007) who approximate the size of the disk on the sky using a boundary between the optically thick and thin disk regions, which then leads to the expectation that good fits fall along a relation where $\log \rho_{0}$ is proportional to $n$.

\section{Discussion}

It is useful to compare our best-fit disk model for $\chi$ Oph to previous results of Waters (1986) who modeled the IRAS IR excesses of a large sample of Be stars with disk models with fixed opening angles of $15^{\circ}$ and where the density varied only with the distance from the stellar center, $r$, in the form of

$$
\rho(r)=\rho_{0}^{\prime}\left(r / R_{\star}\right)^{-n}
$$


Assuming an isothermal disk with a temperature of $18,000 \mathrm{~K}$, a temperature that is considerably larger than the $\sim 10,400 \mathrm{~K}$ density-weighted average temperature of our best-fit model, Waters (1986) found a radial power-law index $n$ of 2.4 and a density at the base of the disk of $\log \rho_{0}=-11.4$. These results compare well to our results of $n=2.5$ and $\log \rho_{0}=-10.7$, even though the disk-density models used in the two studies are not the same. The smaller base disk density found by Waters (1986) is likely the result of the fact that his density distribution is constant along radial arcs over the entire opening angle of the disk which places more material in the disk as compared to our models.

Porter (1999) modeled the circumstellar regions of Be stars as isothermal viscous disks and predicted $n$ of $7 / 2$. Viscous models that drop the assumption of an isothermal disk can find a range of $n$ values as the disk fills or empties (Jones et al. 2008). Porter (1999) also analyzed the IR excess of $\chi$ Oph using the data of Waters (1986), but with a disk model density essentially equivalent to ours. In the case of an isothermal disk (also set to $18,000 \mathrm{~K})$, Porter (1999) found an index $n$ of 2.2 with $\log \rho_{0}=-11.2$; in the case of a disk in which the density-averaged temperature was allowed to vary with radial distance as a power-law, Porter (1999) obtained $n=1.9$ and $\log \left(\rho_{0}\right)=-11.2$. Porter indicates that the non-isothermal disk formally fits the IR data best, but that the improvement is not large.

In addition to the comparison between model and observed $\mathrm{H} \alpha$ interferometric visibilities and the $\mathrm{H} \alpha$ line profile, it is also instructive to compare the predicted IR excess of our best-fit model to the IR observations reported in the literature. Figure 8 illustrates the comparison between the SED of our best-fit model and the visual and IR observations from Waters (1986). The overall agreement is quite reasonable, especially considering that the model was not fit to the SED data, although the observations in the $1-10 \mu \mathrm{m}$ region fall below the model. We should also point out that variability between the observations used by Waters (1986) and our $2006 \mathrm{H} \alpha$ observations (interferometric and spectroscopic) cannot be ruled out. For example, Banerjee et al. (2000) obtained the $\mathrm{H} \alpha$ profile for $\chi$ Oph in 1998 and reported an equivalent width of approximately $-3.6 \mathrm{~nm}$, as compared to the $-7.1 \pm 0.2 \mathrm{~nm}$ found in the present work for the 2006 epoch (recall Fig. 3). Hence, the $\mathrm{H} \alpha$ emission of $\chi \mathrm{Oph}$ has approximately doubled in 8 years, and assuming that the $\mathrm{H} \alpha$ emission was similarly weaker at the time of observations of Waters (1986) we can then expect our best-fit model to over-predict IR excess as compared to Waters' results. For this reason, we feel that our model cannot be improved using the IR observations that were acquired using the IRAS satellite in the early 1980's. 


\section{Summary and Future Work}

In this paper we presented the study of the circumstellar disk of the Be star $\chi$ Oph, based on the approach of combining high-spatial resolution interferometric observations with numerical disk models requiring only a few input parameters. Using the interferometric data we were also able to determine that the $\mathrm{H} \alpha$-emitting disk can be fit by a circular symmetric

Gaussian with FWHM diameter of $3.46 \pm 0.07$ mas. Using the code BEDISK (Sigut \& Jones 2007) we created a grid of models with a range of inner edge densities $\left(\rho_{0}\right)$ and equatorial plane density distribution fall-off rates $(n)$, while keeping the mass, radius and effective temperature of the central star fixed. These models were used to create synthetic $\mathrm{H} \alpha$ images by adding the emission line and continuum flux, including the contribution from the central star, in a $15 \mathrm{~nm}$ spectral region around $\mathrm{H} \alpha$, similar to that of the $\mathrm{H} \alpha$ interferometric channel. These images were then Fourier transformed and compared directly to the interferometric observations, resulting in the best-fit model with $n=2.5$ and $\rho_{0}=2.0 \times 10^{-11} \mathrm{~g} \mathrm{~cm}^{-3}$. The best-fit disk model was also used to calculate a synthetic $\mathrm{H} \alpha$ line profile and spectral energy distribution of this system over $0.1-100 \mu \mathrm{m}$, which show good agreement with the observed line profile and the photometric observations published in the literature.

The technique demonstrated here, of combining models with interferometric observations, demonstrates a new and independent method for obtaining properties of circumstellar disks of Be stars. This is possible because the density distribution in the disk is strongly affected by the value of the disk density at the stellar surface, $\rho_{0}$, and to a lesser extent by $n$. Variations in $\rho_{0}$ and $n$ result in dramatic changes in the thermal structure of the disk. Ultimately, the density distribution and the resulting thermal structure directly affect the $\mathrm{H} \alpha$ flux predicted by the theoretical models, which in turn can be constrained by observations that spatially resolve the region. In the future, we plan to investigate more sophisticated forms of the model density distribution within Be star disks, such as rotating density perturbation models (see for example Wisniewski et al. 2007), and density distributions that are consistent with hydrodynamic simulations.

The Navy Prototype Optical Interferometer is a joint project of the Naval Research Laboratory and the U.S. Naval Observatory, in cooperation with Lowell Observatory, and is funded by the Office of Naval Research and the Oceanographer of the Navy. We thank Doug Gies for the very helpful suggestions on how to improve this manuscript. C. T. thanks Lowell Observatory for the generous telescope time allocation on the John S. Hall Telescope. C. T. would also like to thank Erika Grundstrom for useful discussions and thanks Nick Melena who contributed to the reductions of interferometric observations. C. E. J. and T. A. A. S. would like to acknowledge support from the Natural Sciences and Engineering 
Research Council of Canada.

Facilities: NPOI, LO:42in

\section{REFERENCES}

Abt, H. A., \& Levy, S. G. 1978, ApJS, 36, 241

Banerjee, D. P. K., Rawat, S. D., \& Janardhan, P. 2000, A\&AS 147, 229

Barklem, P. S., \& Piskunov, N. 2003, Modeling of Stellar Atmospheres, 210, 28P

Carciofi, A. C., \& Bjorkman, J. E. 2006, ApJ, 639, 1081

Coté, J., \& Waters, L. B. F. M. 1987, A\&A, 176, 93

Cox, A. N. 2000, Allen's astrophysical quantities, 4th ed. Publisher: New York: AIP Press; Springer, 2000

de Jager, C., \& Nieuwenhuijzen, H. 1987, A\&A, 177, 217

Dougherty, S.M. et al., 1994, A\&A 290, 609

Frémat, Y., Zorec, J., Hubert, A.-M., \& Floquet, M. 2005, A\&A, 440, 305

Gehrz, R. D., Hackwell, J. A., \& Jones, T. W. 1974, ApJ, 191, 675

Gies, D. R., et al. 2007, ApJ, 654, 527

Goraya, P. S. 1984, A\&A, 138, 19

Grundstrom, E. D., \& Gies, D. R. 2006, ApJ, 651, L53

Hall, J. C., Fulton, E. E., Huenemoerder, D. P., Welty, A. D., \& Neff, J. E. 1994, PASP, 106,315

Hanbury Brown, R., Davis, J., \& Allen, L. R. 1974, MNRAS, 167, 121

Harmanec, P. 1987, Bulletin of the Astronomical Institutes of Czechoslovakia, 38, 283

Hummel, C. A., Mozurkewich, D., Armstrong, J. T., Hajian, a. R., Elias II, N. M., \& Hutter, D. J. 1998, AJ, 116, 2536

Hummel, W., in The Be Phenomena in Early-Type Stars, M. A. Smith, H. F. Henrichs, \& J. Fabregat (eds), 2000, ASP Conf. Ser. 214, 396 
Hummel, W., \& Vrancken, M 2000, A\&A, 359, 1075

Hummel, C. A., et al. 2003a, AJ, 125, 2630

Hutter, D. J., Benson, J. A., Zavala, R. T., Johnston, K. J., Pauls, T. A., Hummel, C. A., \& Armstrong, J. T. 2004, Proc. SPIE, 5491, 73

Jones, C. E., Sigut, T. A. A., \& Marlborough, J. M. 2004, MNRAS, 352, 841

Jones, C. E., Sigut, T. A. A., \& Porter, J. M. 2008, MNRAS, 386, 1922

Kastner, J. H., \& Mazzali, P. A. 1989, A\&A, 210, 295

Levato, H., Malaroda, S., Morrell, N., \& Solivella, G. 1987, ApJS, 64, 487

Marlborough, J. M. 1969, ApJ, 156, 135

Meilland, A., et al. 2007, A\&A, 464, 59

Millar, C. E., \& Marlborough, J. M. 1998, ApJ, 494, 715, MM

Millar, C. E., \& Marlborough, J. M. 1999, ApJ, 526, 400

Okazaki, A. T. 2007, Active OB-Stars: Laboratories for Stellare and Circumstellar Physics, Edited by S. Stefl, S. P. Owocki, and A. T. Okazaki., 361, 230

Olson, G. L., \& Kunasz, P. B. 1987, Journal of Quantitative Spectroscopy and Radiative Transfer, 38, 325

Perryman, M. A. C., et al. 1997, A\&A, 323, L49

Porter, J. M. 1996, MNRAS, 280, L31

Porter, J. M. 1999, A\&A, 348, 512

Quirrenbach, A., et al. 1997, ApJ, 479, 477

Rybicki, G. B., \& Hummer, D. G. 1994, A\&A, 290, 553

Sigut, T. A. A. \& Jones, C. E. 2007, ApJ, 668, 481

Sigut, T. A. A., McGill, M. A., \& Jones, C. E. 2007, ApJ, submitted

Tycner, C., Hajian, A. R., Mozurkewich, D., Armstrong, J. T., Benson, J. A., Gilbreath, G. C., Hutter, D. J., Pauls, T. A., \& Lester, J. B. 2003, AJ, 125, 3378 
Tycner, C. et al. 2005, ApJ, 624, 359

Tycner, C., Benson, J. A., Hutter, D. J., Schmitt, H. R., \& Zavala, R. T. 2006a, Proc. SPIE, 6268,49

Tycner, C., et al. 2006b, AJ, 131, 2710

Waters, L. B. F. M. 1986 A\&A, 162, 121

Waters, L. B. F. M., Cote, J., \& Lamers, H. J. G. L. M. 1987, A\&A, 185, 206

Wisniewski, J. P., Kowalski, A. F., Bjorkman, K. S., Bjorkman, J. E., \& Carciofi, A. C. 2007, ApJ, 656, L21

Wood K., Bjorkman K. S., \& Bjorkman J. E. 1997, ApJ, 477, 926

Zorec, J., Frémat, Y., \& Cidale, L. 2005, A\&A, 441, 235 
Table 1: NPOI OBservations of $\chi$ OPH

\begin{tabular}{|c|c|c|}
\hline UT Date & \# of Scans & \# of Baselines \\
\hline 2006 June 11 & 5 & 5 \\
\hline 2006 June 13 & 4 & 5 \\
\hline 2006 June 17 & 6 & 5 \\
\hline 2006 June 18 & 7 & 5 \\
\hline
\end{tabular}

Table 2: Calibrated H $\alpha$ Squared Visibilities of $\chi$ OpH

\begin{tabular}{|c|c|c|c|c|}
\hline $\begin{array}{c}\text { Julian Date } \\
(\mathrm{JD}-2,450,000)\end{array}$ & $\begin{array}{r}\text { Spatial Frequency } u \\
\left(10^{6} \text { cycles/radian }\right)\end{array}$ & $\begin{array}{r}\text { Spatial Frequency } v \\
\left(10^{6} \text { cycles/radian }\right)\end{array}$ & $V_{\mathrm{H} \alpha}^{2}$ & Baseline $^{\dagger}$ \\
\hline 3897.785 & 27.003 & -7.122 & $0.749 \pm 0.048$ & $\mathrm{AC}-\mathrm{AE}$ \\
\hline 3897.785 & 64.880 & 23.478 & $0.534 \pm 0.032$ & W7-AC \\
\hline 3897.785 & 91.883 & 16.357 & $0.502 \pm 0.023$ & W7-AE \\
\hline 3897.785 & -29.723 & -8.345 & $0.688 \pm 0.061$ & AC-AW \\
\hline 3897.785 & 64.989 & 23.518 & $0.519 \pm 0.027$ & W7-AC \\
\hline 3897.785 & 35.266 & 15.173 & $0.680 \pm 0.030$ & W7-AW \\
\hline 3897.801 & 27.324 & -7.970 & $0.696 \pm 0.042$ & $\mathrm{AC}-\mathrm{AE}$ \\
\hline 3897.801 & 61.798 & 21.501 & $0.548 \pm 0.045$ & W7-AC \\
\hline 3897.801 & 89.123 & 13.531 & $0.520 \pm 0.026$ & W7-AE \\
\hline 3897.801 & -28.537 & -7.436 & $0.724 \pm 0.043$ & AC-AW \\
\hline 3897.801 & 61.902 & 21.537 & $0.511 \pm 0.027$ & W7-AC \\
\hline 3897.801 & 33.365 & 14.101 & $0.681 \pm 0.037$ & W7-AW \\
\hline
\end{tabular}

Note. - Table 2 is published in its entirety in the electronic edition of the Astrophysical Journal. $\dagger$ There are 6 baselines per scan (same JD) with the W7-AC measured at two output beams with slightly different wavelength scales and thus resulting in slightly different $u$ and $v$ values for the same baseline length. 
Table 3: Model Stellar Parameters

\begin{tabular}{cccl}
\hline \hline Parameter & Symbol & Value & Reference \\
\hline Mass $\left(M_{\odot}\right) \ldots \ldots \ldots \ldots \ldots \ldots \ldots$ & $M_{\star}$ & 10.9 & Cox $(2000)$ \\
Radius $\left(R_{\odot}\right) \ldots \ldots \ldots \ldots \ldots \ldots$ & $R_{\star}$ & 5.7 & Cox $(2000)$ \\
Effective temperature $(\mathrm{K}) \ldots \ldots$ & $T_{\text {eff }}$ & 20,900 & de Jager \& Nieuwenhuiizen $(1987)$ \\
Luminosity $\left(L_{\odot}\right) \ldots \ldots \ldots \ldots \ldots$ & $L_{\star}$ & $5.6 \times 10^{3}$ & calculated $^{\dagger}$ \\
Surface gravity $\ldots \ldots \ldots \ldots \ldots$ & $\log g$ & 4.0 & calculated $^{\ddagger}$ \\
\hline
\end{tabular}

Note. $-{ }^{\dagger} L_{\star}=4 \pi R_{\star}^{2} \sigma T_{\text {eff }}^{4}$ where $\sigma$ is the Stefan-Boltzmann constant. ${ }^{\ddagger} \log g=\log \left(G M_{\star} R_{\star}^{-2}\right)$ where $G$ is the gravitational constant. 


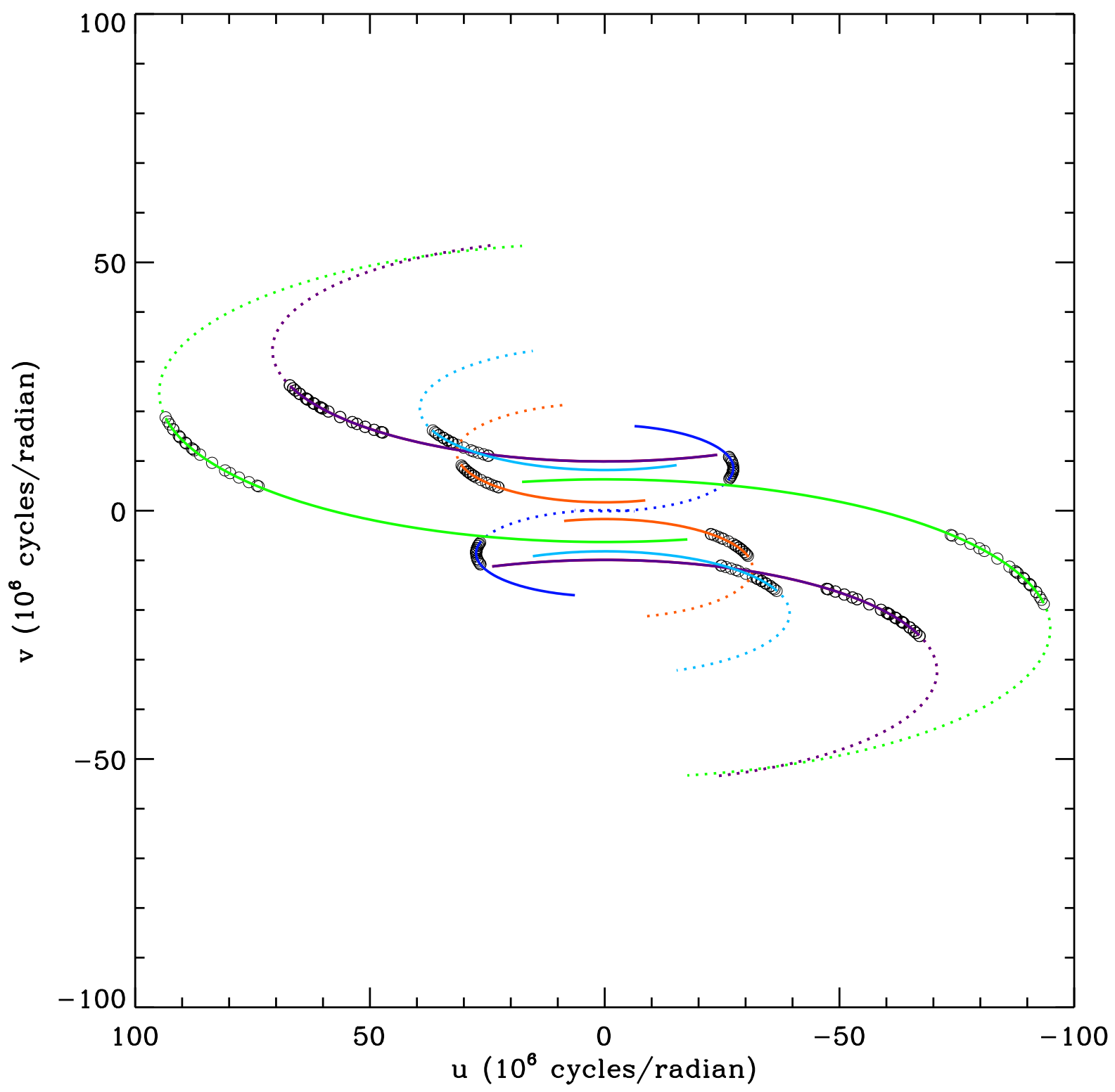

Fig. 1.- The $(u, v)$-plane coverage of the interferometric observations of $\chi$ Oph obtained in the $\mathrm{H} \alpha$ channel with five unique baselines from four nights of observations (circles). The dotted-lines indicate possible coverage from meridian to $6 \mathrm{hr}$ east and the solid-lines indicate possible coverage from the meridian to $6 \mathrm{hr}$ west. 


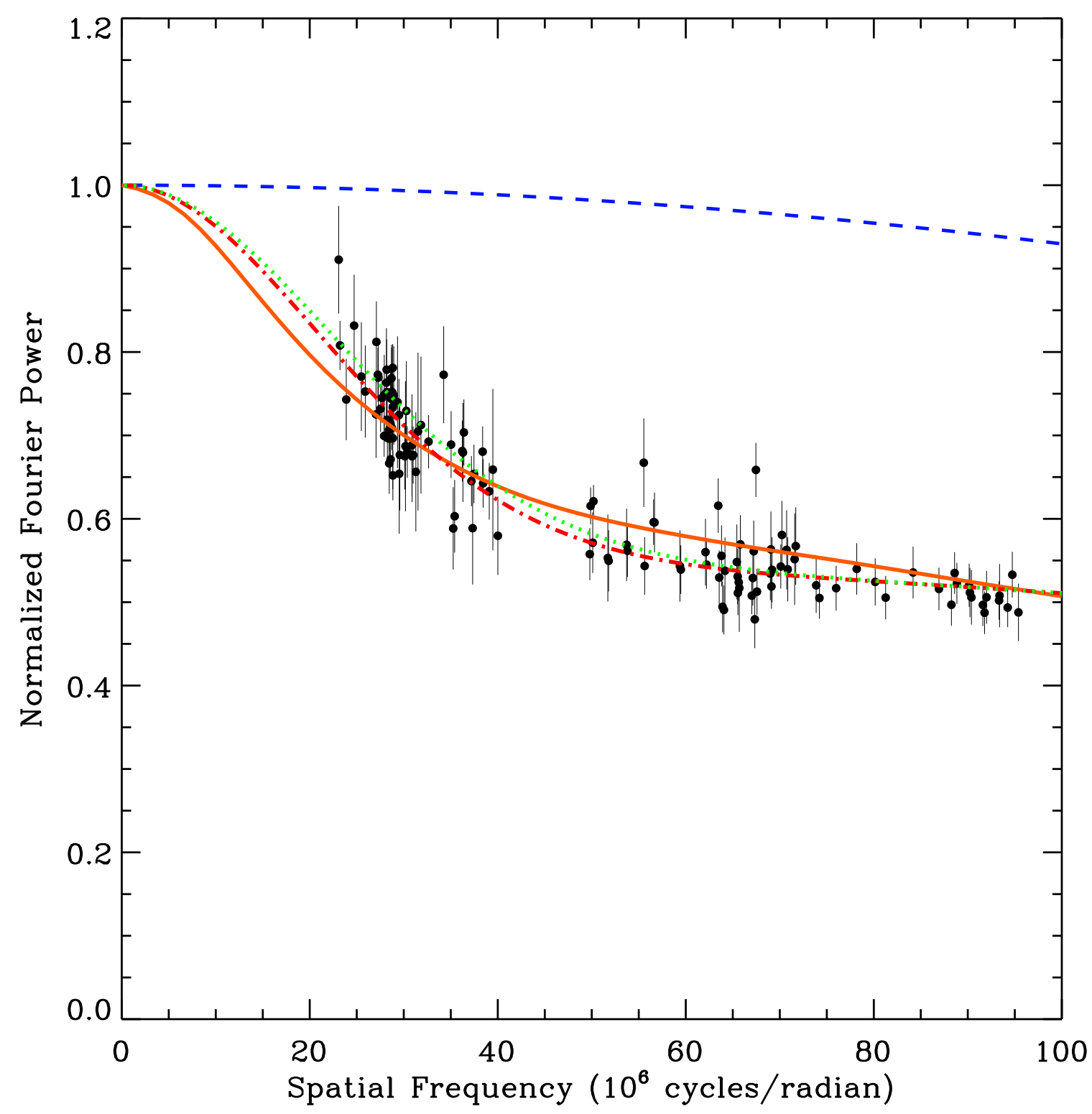

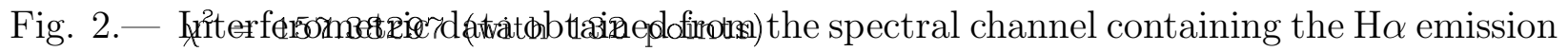
line from the circumstellar disk of $\chi \mathrm{Oph}$. The $0.52 \mathrm{AU}$ diameter (FWHM) circumstellar disk is fully resolved, whereas the central star is assumed to have an uniform disk diameter of 0.35 mas (dashed line). The best-fit model (solid line) obtained by taking the Fourier transform of the synthetic image from Figure 6 is shown along with a circularly symmetric Gaussian model with FWHM diameter of 3.46 mas (dash-dotted line). The effect of an axial ratio of 0.94 on the Gaussian model is also shown (dotted line). 


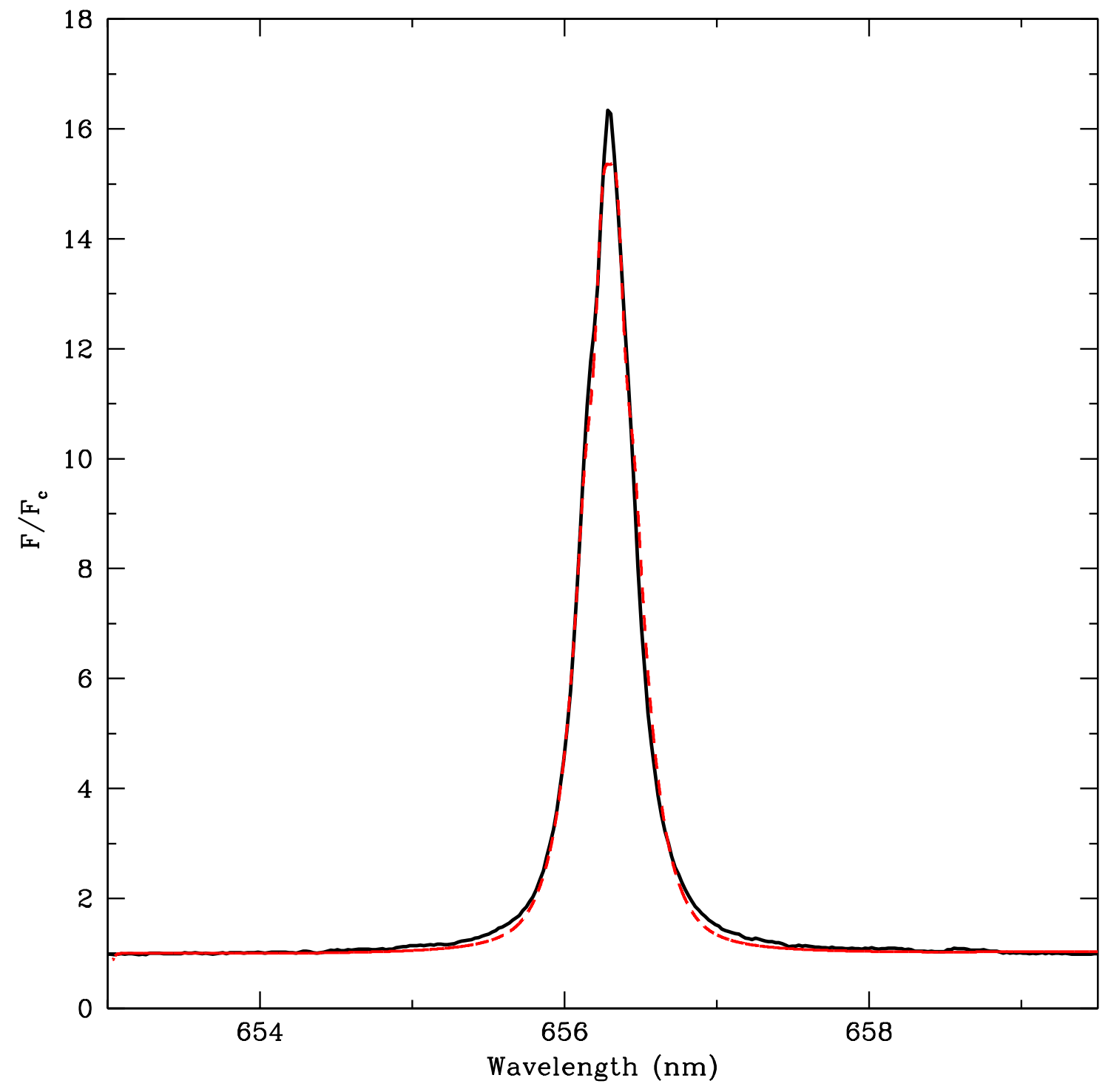

Fig. 3.- The $\mathrm{H} \alpha$ emission line of $\chi$ Oph observed on 2006 June 10 (solid-line) and the synthetic $\mathrm{H} \alpha$ profile calculated from the best-fit disk model (dashed-line). The model profile has been obtained for an inclination angle $i$ of $20^{\circ}$ and has been broadened by a Gaussian kernel to match the resolving power $(R=10,000)$ of the spectroscopic observations. The observed $\mathrm{H} \alpha$ profile has an EW of $-7.1 \pm 0.2 \mathrm{~nm}$ and the theoretical profile has EW of $-6.6 \mathrm{~nm}$. 


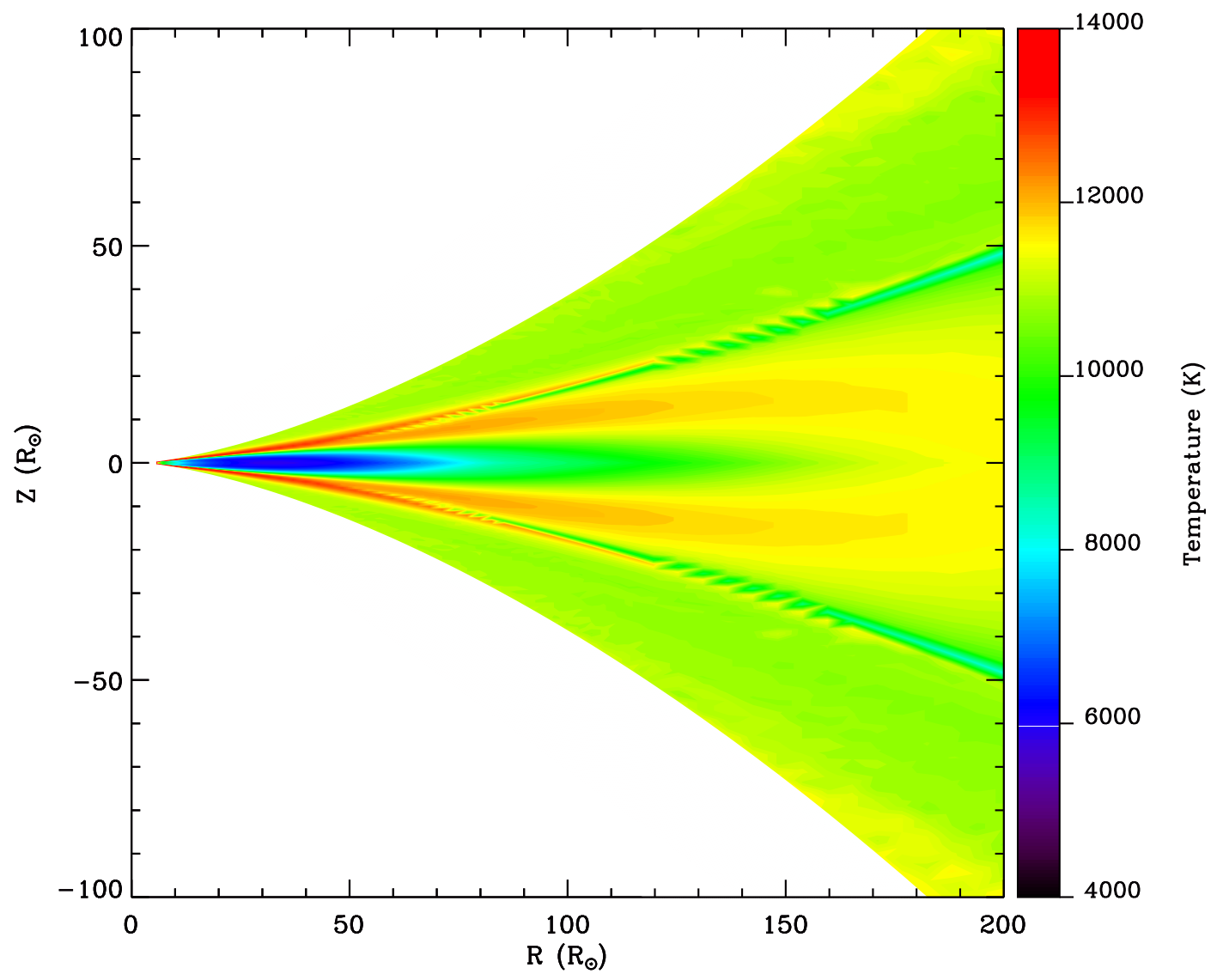

Fig. 4.- The thermal structure of the disk model of $\chi$ Oph obtained using $n=2.5$, $\rho_{0}=2.0 \times 10^{-11} \mathrm{~g} \mathrm{~cm}^{-3}$ and the stellar parameters listed in Table 3 , The thermal structure is shown as a function of radial distance $(R)$ from the center of the star and the vertical distance $(Z)$ above and below the equatorial plane. 


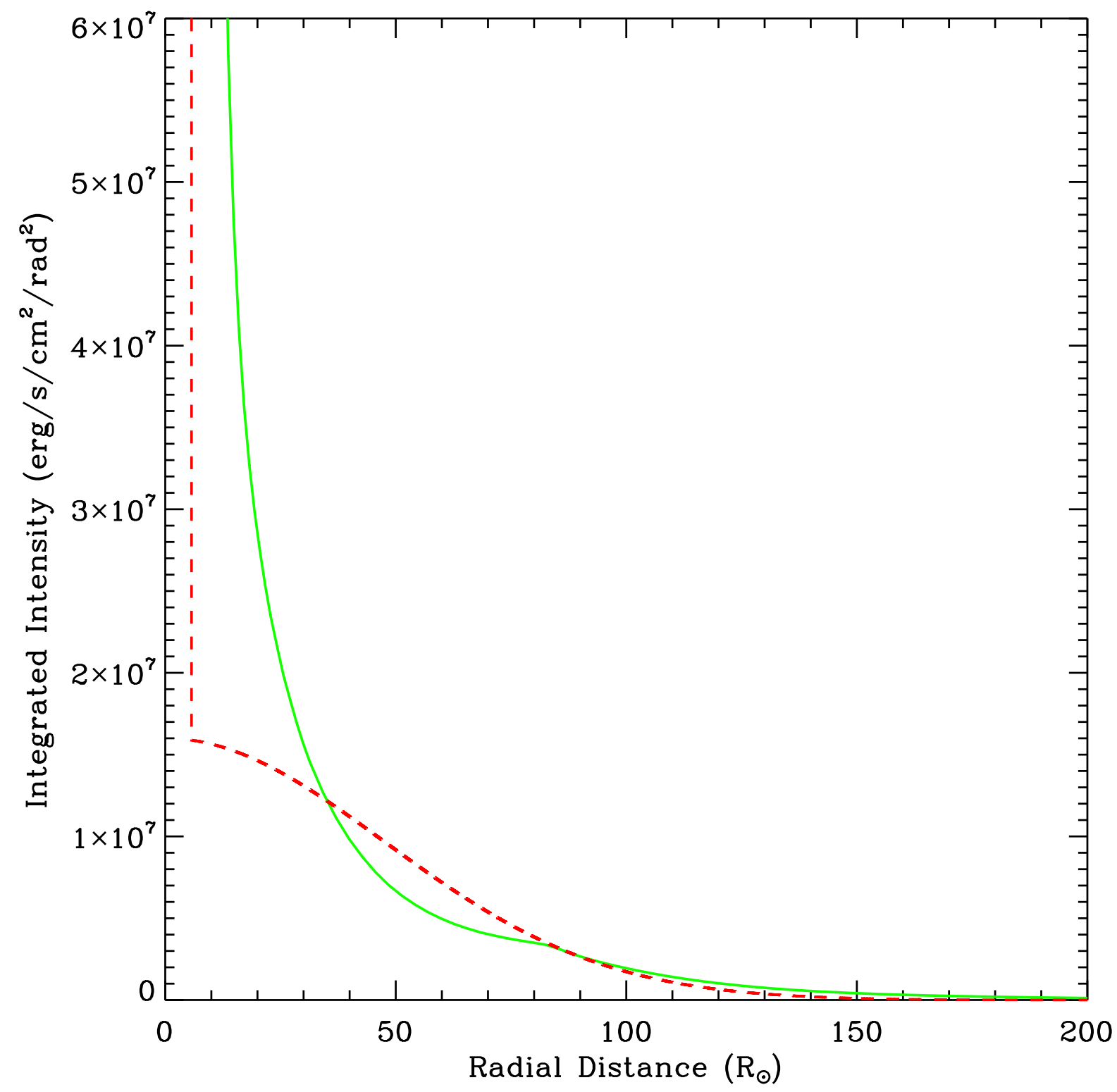

Fig. 5.- Integrated model intensity for the direction normal to the plane of the disk in a $15 \mathrm{~nm}$ wide spectral region centered at $\mathrm{H} \alpha$ (solid line) for the best-fit model of $n=2.5$ and $\rho_{0}=2.0 \times 10^{-11} \mathrm{~g} \mathrm{~cm}^{-3}$. The Gaussian radial intensity distribution that reproduces the interferometric signature in Fig. 2 is also shown (dashed line). 


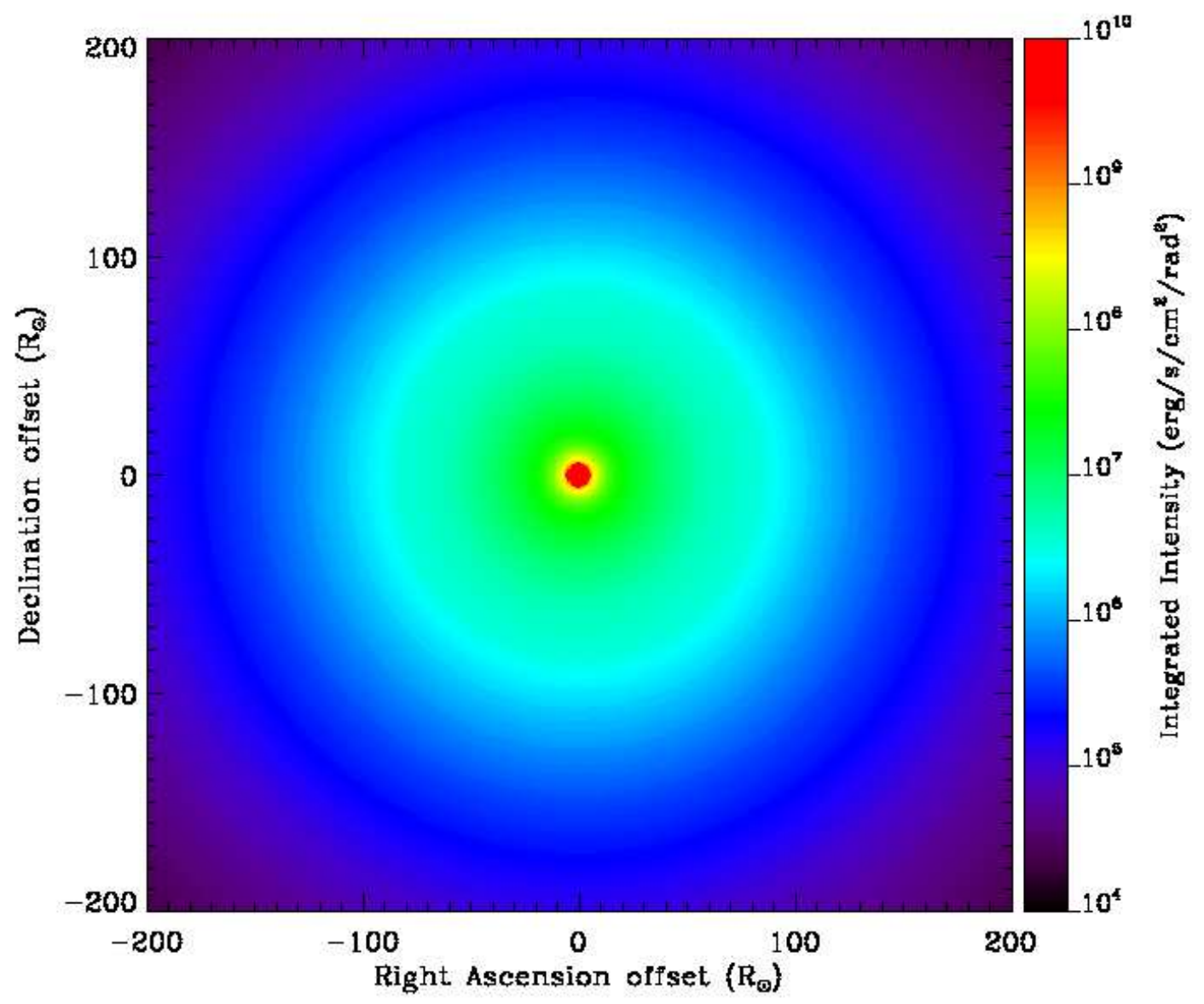

Fig. 6. - Synthetic image of the best-fit disk model viewed pole on. Only intensities in a $15 \mathrm{~nm}$ spectral window centered at $\mathrm{H} \alpha$ emission line were used to construct this synthetic image. The surface brightness of the central star is derived using the stellar atmosphere model integrated over the same $15 \mathrm{~nm}$ spectral window as used to calculate the net intensity from the disk. 


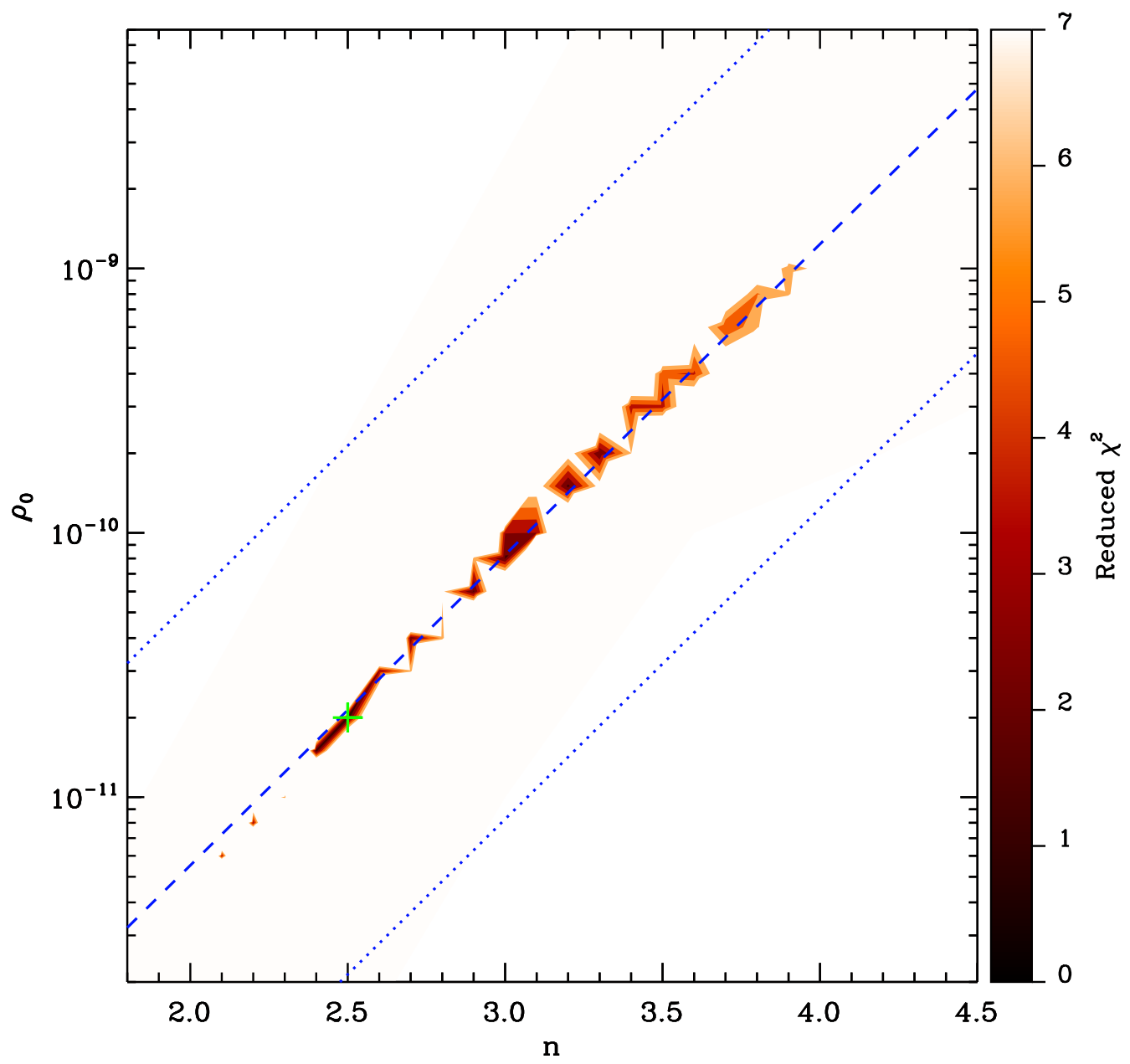

Fig. 7.- Contour plot showing the reduced $\chi^{2}$ value as a function of $\rho_{0}\left(\mathrm{~g} \mathrm{~cm}^{-3}\right)$ and $n$. Only a region of reduced $\chi^{2}<7$ is shown and the best-fit model with reduced $\chi^{2}$ of 1.17 is marked with a plus sign. Some of the gaps in the linear trend are caused by the grid spacing and the limitations of the contour plotting routine dealing with unequal grid spacing. The approximate boundary of the region of parameter space sampled by our models is also indicated (dotted lines), along with a linear fit (dashed line) to the models with reduced $\chi^{2}<7$ in the $\log \rho_{0}$ and $n$ plane $($ see $\S 5.2)$. 


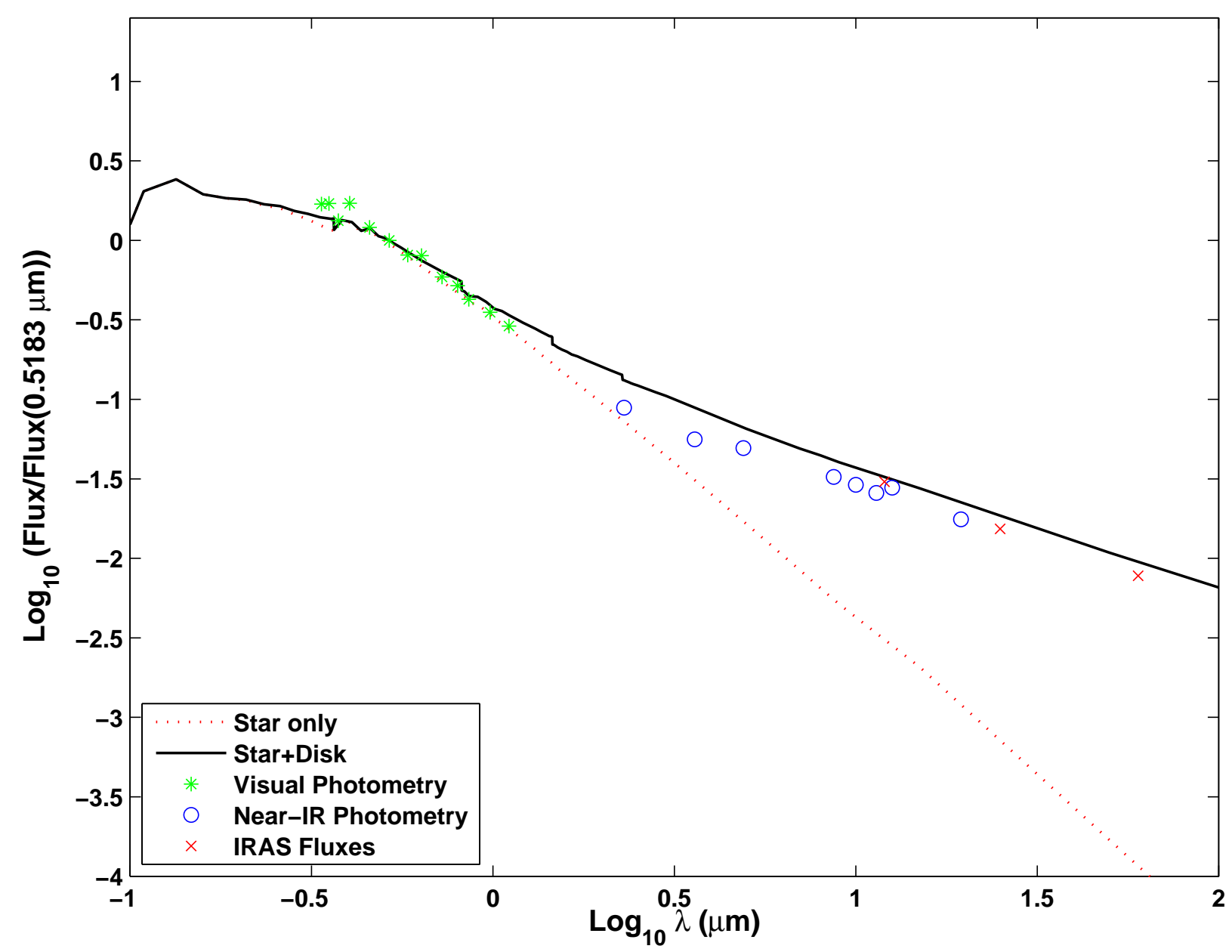

Fig. 8. - The spectral energy distribution (SED) of our best-fit model corresponding to $n=2.5$ and $\rho_{0}=2.0 \times 10^{-11} \mathrm{~g} \mathrm{~cm}^{-3}$ (solid-line), which consists of the sum of the stellar contribution (dotted-line) and IR excess produced by the circumstellar disk. The model SED is compared to visual, near-IR and far-IR observations reported in literature (see Waters 1986, and references therein). 